\title{
Automated Online Measurement of Particle Size Distribution using 3D Range Data
}

\author{
Matthew J. Thurley \\ Luleå University of Technology, Luleå, SE-97187 Sweden (e-mail: \\ matthew.thurley@ltu.se).
}

\begin{abstract}
Fully automated online measurement of the size distribution of limestone fragments on conveyor belt is presented based on 3D range data collected every minute during 13 hours of production. The research establishes the necessary measurement technology to facilitate automatic control of particle breaking or aggregating processes to improve both energy efficiency and product quality. Techniques are presented covering; sizing of fragments, determination of non-overlapped and overlapped fragments, and mapping of sizing results to distributions comparable to sieving. Detailed variations in the product sieve size are shown with an abrupt change when the size range of the limestone fragments was changed.
\end{abstract}

Keywords: image segmentation, particle size measurement, range data, classification

\section{INTRODUCTION}

In the mining and aggregate industries a great deal of effort goes into measuring or estimating the size distribution of particulate material. One reason is that suppliers of particulate material are typically paid to supply a specific size range of material. For both industries there is also a key desire for energy efficiency and size quality that is relevant in both crushing and aggregating processes. In addition, blasting and caving are very cost effective methods of rock breakage, but they are not processes that are easily quantified.

Mine and quarry operators want to measure the particle sizing results of all of these activities but sieving is typically impractical as a routine assessment tool due to slow feedback, inconsistent measurement, and time consuming interruption.

As a result there is an opportunity for online, non-contact, fully automated machine vision systems for measurement of particle size that can provide the necessary accuracy and fast feedback to facilitate process control and allow automatic control optimisations for both product size and energy efficiency. This is relevant to a vast range of processes that modify particle size such as blasting, crushing, pelletising, and in ovens and kilns.

There are however, a number of sources of error relevant to techniques that measure only what is visible on the surface of a pile as follows;

Segregation and grouping error, more generally known as the brazil nut effect (Rosato et al., 1987), describes the tendency of the pile to separate into groups of similarly sized particles. It is caused by vibration or motion (for example as rocks are transported by truck or conveyor) with large particles being moved to the surface. It is advisable to measure at a point early on the conveyor before the material has been subjected to excessive vibration and segregation.
Overlapped particle error, describes the fact that many particles are overlapped (see figure 1) and only partially visible and a large bias to the smaller size classes results if they are treated as small non-overlapped and sized using only their visible profile. This error can be overcome in piles of particulate material using classification algorithms based on 3D range data (Thurley and Ng, 2008).

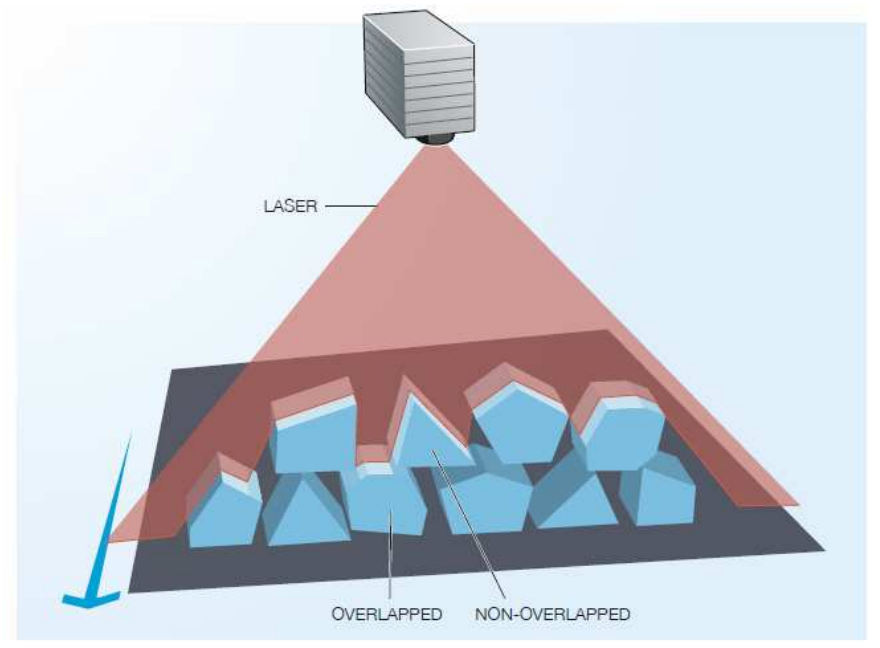

Fig. 1. Illustration of overlapped and non-overlapped particles.

Capturing error, describes the varying probability based on size, that a particle will appear on the surface of the pile. In simple terms, the larger a particle is, the more likely one is to be able to see some part of it on the surface. For example, if a single particle is as large as the height of the pile of material, then it will always be visible, whereas a very fine particle is almost certainly not visible. Thurley (2002) has explored capturing error in laboratory rock piles but it remains a source of error in this application.

Profile error, describes the fact that only one side (a profile) of an entirely visible particle can be seen making 
if difficult to estimate the particles size. However, if the particle is not overlapped, best-fit-rectangle (Wang, 2006) has been demonstrated as a suitable feature for size classification based on the visible profile, that correlates to sieve size.

In addition to these errors, we note that size measurement using imaging identifies how many particles are observed of various size classes, but manual sieving measures the weight of particles in each size class. Therefore it is necessary to have a method of mapping from numbers of particles to weight of particles in order to provide a measurement of size that industry understands and can use. A technique based on physical sampling and sieving results is used in this work and explained in detail subsequently.

Particle size measurement using vision has been the subject of research and development for over 25 years (Carlsson and Nyberg, 1983) with a legacy of predominantly photographic based systems with widely varying degrees of success and no general solution available on the market.

Photographic based 2D imaging systems are subject to bias due to uneven lighting conditions, excessive shadowing, color and texture variation in the material, and lack of distinction between overlapped and non-overlapped fragments.

In their review of a commercial photographic based 2D system Potts and Ouchterlony (2005, pg. vi, viii) report that for their application the system erroneously assumes the resultant size distribution is unimodal and they conclude by expressing strong reservations saying $2 \mathrm{D}$ "imaging has a certain but limited usefulness when measuring the fragment size distribution in a muckpile or from a belt in an accurate way. It could probably detect rough tendencies in fragmentation variations, if the lighting conditions do not vary too much, and if cover glasses for camera lenses are kept clean".

There are a number of publications relating to $3 \mathrm{D}$ size measurement, Noy (2006, rocks), Frydendal and Jones (1998, sugar beets), Kim et al. (2003, river rock) Lee et al. (2005). However, Frydendal and Jones (1998), and the presenting author Thurley and $\mathrm{Ng}$ (2008) are the only publications (2D or $3 \mathrm{D}$ ) to remove the bias resulting from overlapped particles. For conveyor belt applications Kim et al. (2003) and Lee et al. (2005) recommend installing a mechanical vibration feeder to separate rocks and prevent particle overlap. However, such an addition to the plant is typically impractical and for the examination of rocks in buckets (Thurley, 2009), there is no other option than to account for overlapped and non-overlapped fragments. Frydendal and Jones (1998) used graph theory and average region height to determine the entirely visible sugar beets but this relied on the regular shape and size of the beets. Only the presenting author has made this distinction between overlapped and non-overlapped particles using the advantages of $3 \mathrm{D}$ range data and in a manner that does not presume constraints on size or shape (Thurley and $\mathrm{Ng}, 2008$ ).

We use an industrial measurement system on conveyor belt based on laser triangulation (a projected laser line and camera triangulation) collecting highly accurate 3D profiles of the laser line at about $3000 \mathrm{~Hz}$. This high speed ensures we have a high density of $3 \mathrm{D}$ point data at a spacing between consecutive points in the direction of the belt of approximately $1 \mathrm{~mm}$. The imaging system is installed at a limestone quarry on the conveyor belt used for ship loading and measures the material on the belt during loading every minute.

The computational speed of the analysis process is approximately 53 seconds on a $2 \mathrm{GHz}$ Mobile Pentium 4 processing a data set of $590,000+3 \mathrm{D}$ points ( $2 \mathrm{~m}$ long section of the belt). Furthermore, multi-core CPUs effectively offer a linear increase in the rate at which data could be sampled from the conveyor and processed. Further advances in both algorithmic efficiency and hardware are both available to improve computational time for rapid automatic control applications.

\section{RESEARCH BACKGROUND}

The presented research builds upon a series of achievements and research developed on both laboratory rock piles and industrial application.

We have previously implemented an industrial measurement system on conveyor belt for iron ore pellets (Thurley and Andersson, 2007) using the same laser triangulation measurement technology. The high speed camera system ensures we have a high density of $3 \mathrm{D}$ point data at a spacing between consecutive points in the direction of the belt of approximately $0.5 \mathrm{~mm}$. This high data density has at least two advantages. Firstly it allows us to detect small sliver regions or crescent-like regions of overlapped particles and ensure that they are not merged into other regions. And secondly, it has ensured that we could detect a very high resolution when it came to measuring the size of each iron ore pellet allowing a size distribution with very fine spacing of $5,8,9,10,11,12.5,13,14$, and $16+\mathrm{mm}$ size classes.

One of the key criteria for particle size measurement is therefore high data density as it defines the capacity to detect small overlapped particles, the lower limit on particle size that can be reliably detected, and the resolution of size classes detectable.

Another criteria is the computational speed of the analysis. It is generally desirable in a conveyor belt situation to sample as frequently as possible, but a sufficient sampling rate depends on the specific application. In the presented application for limestone both the lower limit of particle size (about $10 \mathrm{~mm}$ ) and the response time to deviations in size during ship loading (about 5 minutes) are well within the computational speed and resolution capabilities of the system.

In addition we have performed a demonstration project for size measurement of rocks in underground LHD excavator buckets Thurley (2009). A 3D vision system based on laser scanners was installed on the tunnel roof in a production area of an underground iron ore mine with 3D surface data of the bucket contents being collected as the LHD unit passes beneath. The project successfully demonstrated fragmentation measurement of the rocks in the bucket, identifying overlapped rocks, non-overlapped rocks, areas of fine material, estimating the sieve size of the visible 
rocks only, and the calculating the proportion of the surface that was identified as fine particles below the observable resolution of the laser scanner.

\section{ANALYSIS OF 3D RANGE DATA}

\subsection{Segmentation}

The first step is to perform the detailed image segmentation to identify the individual rock fragments. These techniques have been applied to laboratory rock piles (Thurley and $\mathrm{Ng}, 2005$ ) and in an industrial pellet measurement system (Thurley and Andersson, 2007) and were adapted to this application. The technique is predominantly based on morphological image processing, based largely on various edge detection techniques to facilitate seed formation for the watershed segmentation algorithm.

Figure 2 shows a closeup image of rocks on the conveyor and the automated segmentation result.

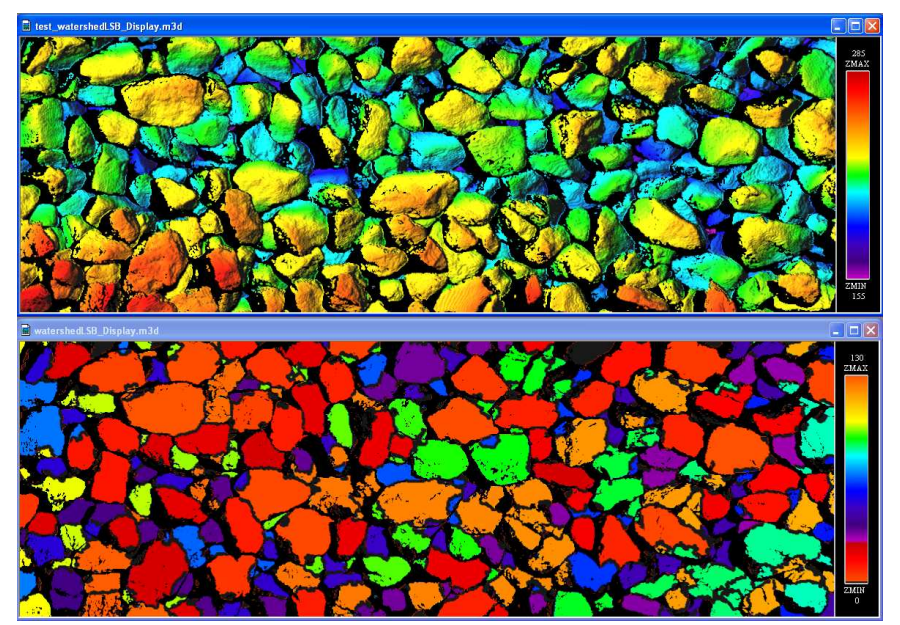

Fig. 2. Rocks on conveyor and automated segmentation for limestone aggregate, $40-70 \mathrm{~mm}$ size range.

\subsection{Non-overlapped Particles}

In order to explain the determination of overlapped and non-overlapped particles it is necessary to introduce a boundary following algorithm developed by Thurley and $\mathrm{Ng}$ (2008) for the purpose of overlapped particle detection.

The algorithm begins with a segmentation of a pile of particulate material and examines each region in the segmentation. For each region in the segmentation we identify a series of prominent points equally spaced around the perimeter of the region.

Looking at figure 3 we see a representation of two regions (one overlapping the other) with a series of prominent points marked for region $R_{i}$.

We then use these points to define small local areas in which we examine the height of the regions in that local area. In the magnified section of figure 3 you can see a circular area about point $g$, we examine this area, denoted $N_{g}$ and calculate the average height of all of the points in the region $R_{i}$ that intersect area $N_{g}$, and we calculate the average height of all of the points in the

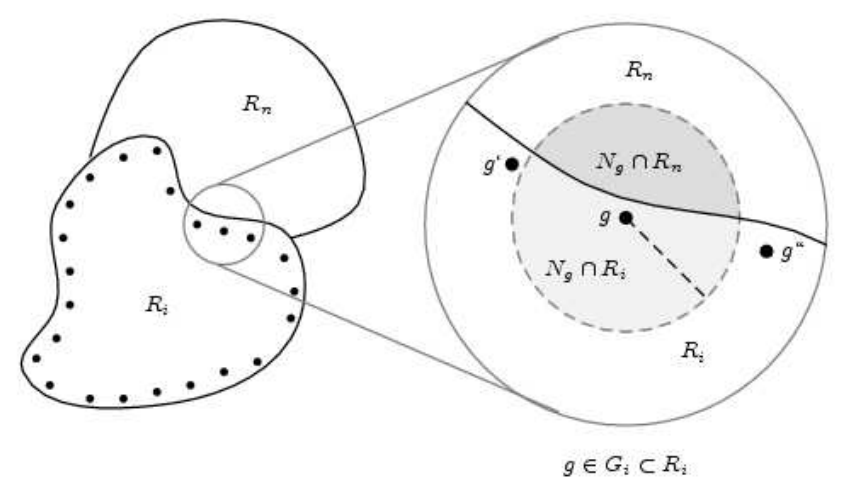

Fig. 3. Boundary following algorithm

region $R_{n}$ that intersect region $N_{g}$. By comparing these two average height values we can evaluate whether in the local neighbourhood of point $g$, region $R_{i}$ is either above or below region $R_{n}$.

This determination is used to determine overlapped/nonoverlapped particles by counting the ratio of points $g$ where region $R_{i}$ is above region $R_{n}$.

This distinction allows us to eliminate misclassification of overlapped rocks are if they were smaller non-overlapped rocks.

We apply an equivalent strategy to that applied on laboratory rock piles (Thurley and Ng, 2008) using the visibility ratio and selecting a decision boundary of 0.65 . Figure 4 shows only the rocks that were classified as non-overlapped from figure 2 .

We will perform sizing on the non-overlapped rocks for which we can make a confident size prediction, and we will ignore overlapped rocks.

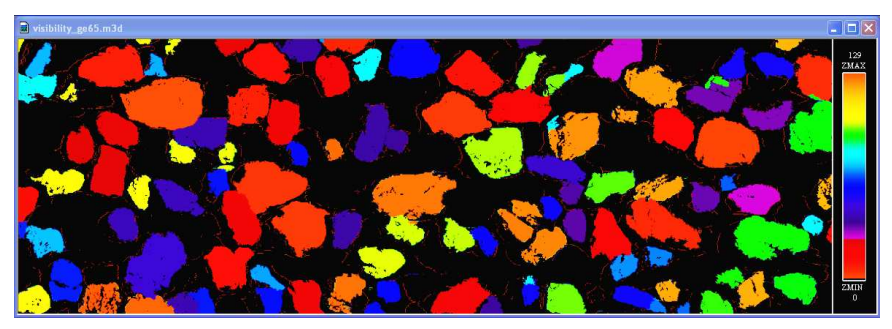

Fig. 4. Non-overlapped rocks automatically identified from figure 2, 40-70 $\mathrm{mm}$ size range.

\subsection{Sizing}

Sizing begins by calculating a feature called best-fitrectangle area for each of the regions identified as nonoverlapped fragments from the segmentation (figure4). The best-fit-rectangle is the rectangle of minimum area that fits around the $2 \mathrm{D}$ projection of each region. We ignore the height component of the points in a region and calculate an arbitrarily rotated best-fit-rectangle. We have used this feature previously (Thurley and Ng, 2008; Thurley and Andersson, 2007, laboratory rock piles, iron ore pellets) and demonstrated its suitability for estimating sieve size of non-overlapped particles.

In order to classify best-fit-rectangle areas into sieve size classes however, we require a series of decision boundaries. 
In effect, thresholds that define which area values separate one size class from another. To determine these values we use some sieving data that corresponds to some of our image analysis results.

During the measurement campaign we collected a 3D data set of the surface of the rock stream every minute for approximately 13 hours of production. In this time we obtained just over $6003 \mathrm{D}$ data measurement sets of limestone on the conveyor belt, and automatically identified and sized over 140,000 rocks. In addition, the mining company obtained some manual samples from the conveyor using a automated sampler, and subsequently sieved these in the laboratory. Table 1 shows these sieve results. What we see is that two distinct product ranges of material were being loaded at different times. Firstly the $40-70 \mathrm{~mm}$ product, then the $20-40 \mathrm{~mm}$ product, before changing back to the $40-70 \mathrm{~mm}$. For a given sieving, most of the material is within the product size range, but there is always some small proportion above and below this range. We also note that the sieve decks were changed between the two different products.

\section{Table 1. Sieving results (cumulative \%)}

\begin{tabular}{|c|c|c|c|c|c|c|}
\hline \multirow{4}{*}{$\begin{array}{c}\text { Sieve } \\
\text { Size } \\
(\mathrm{mm})\end{array}$} & \multicolumn{6}{|c|}{ Product } \\
\hline & \multirow{2}{*}{\multicolumn{6}{|c|}{$\begin{array}{l}20-40 \mathrm{~mm} \\
\quad \text { Sieving Time }\end{array}$}} \\
\hline & & & & & & \\
\hline & $21: 20$ & $23: 05$ & $00: 15$ & $06: 50$ & 09:45 & $11: 40$ \\
\hline$>75$ & 100 & 100 & 100 & 100 & 100 & 100 \\
\hline$<75$ & 100 & 100 & 100 & 100 & 95.2 & 89.9 \\
\hline$<63$ & 77.8 & 100 & 100 & 76.2 & 78.1 & 59.6 \\
\hline$<50$ & 45.9 & 100 & 100 & 40.8 & 40.9 & 28.1 \\
\hline$<45$ & - & 98.7 & 100 & - & - & - \\
\hline$<40$ & 5.83 & 87.1 & 86.8 & 6.56 & 4.79 & 3.65 \\
\hline$<37.5$ & 2.43 & - & - & 2.82 & 1.86 & 1.57 \\
\hline$<31$ & - & 44.2 & 36.3 & - & - & - \\
\hline$<25$ & 0 & 14.6 & 10.6 & 0 & 0 & 0 \\
\hline $12-20$ & 0 & 0.736 & 0.762 & 0 & 0 & 0 \\
\hline
\end{tabular}

We select two data sets from each product range (times 23:05, 00:15, 09:45, and 11:40) and use the sieving data and corresponding image analysis results to calculate decision boundaries for mapping best-fit-rectangle area into sieve size class.

We use the Nelder and Mead (1965) iterative error minimisation process to calculate decision boundaries with minimised error. This optimisation method is available both in Matlab using function fminsearch, or in the statistical package $R$ using function optim which we use here. Given an initial estimate of the decision boundaries we use NelderMead minimisation to produce a final set of boundaries.

The Nelder-Mead optimisation takes an estimate of the decision boundaries and calculates the error at that estimate (we define this error function in more detail in the next section). The optimisation modifies the decision boundaries slightly following a range of internal rules before repeating the process. Changes that reduce the error are kept, while changes that increase the error are typically discarded. We first calculate optimum decision boundaries for each set of sieving data and then calculate the median values as the initial estimates for the Nelder-Mead and generate the error minimised result.

Figure 5 shows the distribution of best-fit-rectangle (BFR) area measurements for the non-overlapped rocks calculated by the system for the two different products from all
$600+3 \mathrm{D}$ measurements. We can clearly see that when the smaller product is on the belt, smaller values of the BFR area are generally calculated. We also show the location of the decision boundaries for each sieve size on the bottom axis as calculated using the Nelder-Mead optimisation.

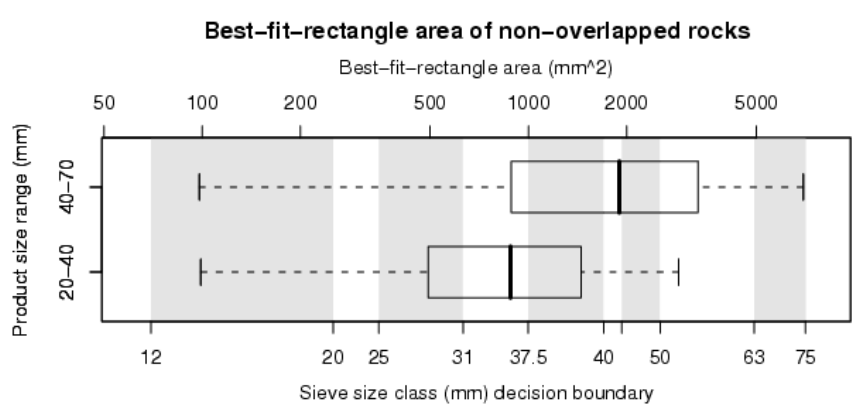

Fig. 5. Best-fit-rectangle areas results for the Nonoverlapped rocks by product size range.

\subsection{Mapping from Number to Weight}

One of the complications for image analysis systems is that they can measure the number of observable particles, and using classification strategies for size can calculate number of particles in each size class. However, what is required is the estimated weight of the material in each size class as this percentage by weight is what is measured by sieving and required by industry. Therefore it is necessary to convert number of particles by size class into an estimated weight by size class. We do this by collecting sieving results and counting the number of particles in each size class in order to determine an average weight of a fragment by size class as shown in table 2 .

\section{Table 2. Mean Particle Weight by Size Class}

\begin{tabular}{llcccccc}
\hline Sieve & Mid & \multicolumn{7}{c}{ Mean Particle Weight $(\mathrm{g})$} \\
Size & Size & \multicolumn{7}{c}{ Time of Sieving } \\
$(\mathrm{mm})$ & $(\mathrm{mm})$ & $21: 20$ & $23: 05$ & $0: 15$ & $6: 50$ & $9: 45$ & $11: 40$ \\
\hline$>75$ & 80 & - & - & - & - & 793 & 719 \\
$63-75$ & 69 & 540 & - & - & 480 & 504 & 509 \\
$50-63$ & 56.5 & 289 & - & - & 276 & 285 & 311 \\
$45-50$ & 47.5 & na & 168 & - & na & na & na \\
$40-50$ & 45 & 164 & na & na & 172 & 172 & 187 \\
$40-45$ & 42.5 & na & 115 & 117 & na & na & na \\
$37.5-40$ & 38.8 & 132 & na & na & 129 & 105 & 148 \\
$31-40$ & 35.5 & na & 73.7 & 72.3 & na & na & na \\
$31-37.5$ & 34.3 & na & na & na & na & na & na \\
$25-37.5$ & 31.3 & 72.5 & na & na & 80.2 & 84.3 & 99.9 \\
$25-31$ & 28 & na & 42.3 & 44.0 & na & na & na \\
$20-25$ & 22.5 & - & 27.1 & 27.6 & - & - & - \\
$12-20$ & 16 & - & 9.21 & 7.98 & - & - & - \\
\hline
\end{tabular}

The immediate thing that one notes from table 2 is that different arrangements of sieve decks were used at different times (for the different products) complicating the capacity to compare and combine the results. As a result we calculate a polynomial of best-fit for the sieve mid-size versus weight. This provides a continuous and consistent estimate of weight for any given size class that we wish to estimate with the imaging.

Based on the data in table 2 we calculate a third order polynomial where $x$ is the mid size of the sieve range and 
the weight is given by $f(x)=0.001208 x^{3}+0.01494 x^{2}+$ $0.5306 x$ with correlation $R^{2}=0.9909$. The graph of table 2 and the best-fit polynomial is not shown due to page limitations.

This mismatch between measuring numbers of particles and correlating against weight affects the Nelder-Mead error minimisation process. As a result we use the weights calculated using the best-fit polynomial within the NelderMead error minimisation. We express this error function as a sum of squares as shown in equation (1). The function uses a normalized weight value derived from the polynomial weight function $f$. The normalized weight provides a weight of 1 for the heaviest size class at a given time $t$, with the lighter weights scaled relatively to this value. The purpose of this normalization is to ensure that heavier product ranges do not dominate the error function. Without this normalization the results for the $40-70 \mathrm{~mm}$ product dominate the optimization effectively ignoring the error for the $20-40 \mathrm{~mm}$ product.

$$
\begin{aligned}
& \epsilon=\sum_{t}\left[\sum_{i=1}^{N}\left[\left(C_{x, t}-S_{x, t}\right) \widehat{W}_{x, t}\right]^{2}\right] \\
& \widehat{W}_{x, t}=\frac{f(x)}{\max _{t}(f)} \\
& t \quad \text { Time of day } \\
& x \quad \text { Sieve size class } \\
& N \quad \text { Number of sieve size classes } \\
& C_{x, t} \quad \text { Calculated value of the size class } x \text { at time } t \\
& S_{x, t} \quad \text { Sieving result for size class } x \text { at time } t \\
& f(x) \quad \text { Weight of size class } x \text { using polynomial weight function } \\
& \max _{t}(f) \quad \text { Weight of heaviest size class at time } t \\
& \widehat{W}_{x, t} \quad \text { Normalized weight }
\end{aligned}
$$

Furthemore, as decision boundaries must be monotonically increasing with size class $x$ (the larger the size class the larger the decision boundary), we must further constrain the error-function by using a penalization factor when the decision boundaries are out of order. Specifically we scale the error by the amount that any consequtive decision boundaries is out of order. Without this constraint the error minimization can generate meaningless out of order results.

Using the optimised Nelder-Mead decision boundaries for determining the sieve size class boundaries (depicted in figure 5) we produce estimates of the sieve size distributions for the 606 measurement sets. The results are presented in figure 7 with the sieve data from table 1 overlayed as colored points.

If we look only at the sieving results from 0:15 and 6:50, and the two imaging mesurements that correspond to these times we can plot a typical log-linear cumulative size distribution curve as shown in figure 6 .

\section{DISCUSSION}

The imaging results (figure 7) are shown to clearly trend in the right direction tracking changes in the material size. Furthermore, in the higher size classes, 50, 63, 75, where decision boundaries could be set for the $40-70 \mathrm{~mm}$ product independantly of the $20-40 \mathrm{~mm}$ product, the imaging results appear to track the sieving results well.
Size Distribution at 0:15 and 6:50

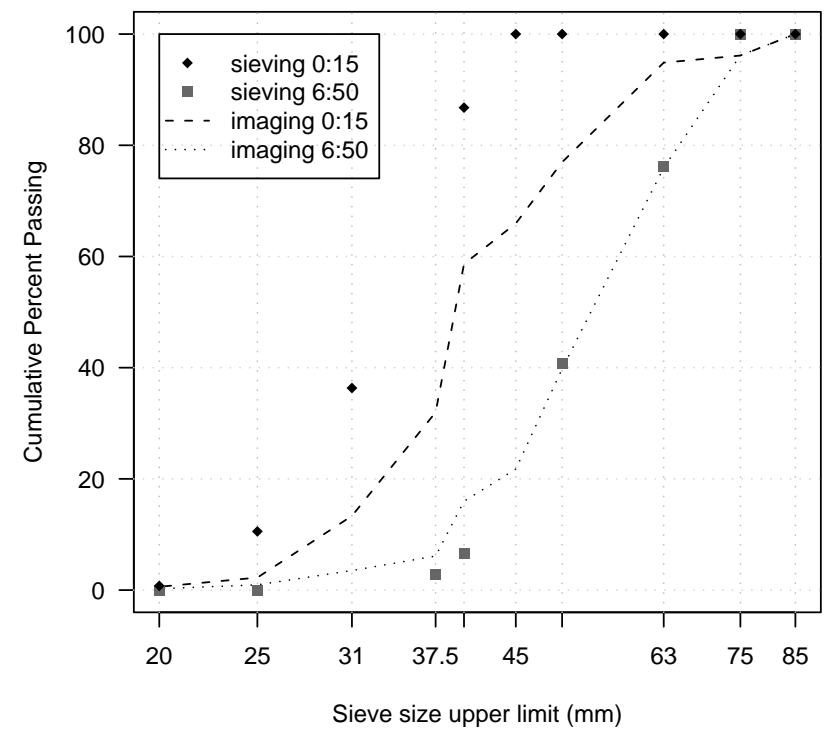

Fig. 6. Imaging and sieving results for 2 measurements and sieve results at $0: 15$ and $6: 50$.

If we examine figure 7 and compare it to the cumulative sieving results in table 1 we can see that the image analysis overestimates the amount of $<40 \mathrm{~mm}$ size class in the 40 $70 \mathrm{~mm}$ product, by about $10 \%$, and underestimates the cumulative amount in the $20-40 \mathrm{~mm}$ product by about $25 \%$.

The key problem is that an average weight by size class will work fine if the fragments are always uniformly distributed across that size class. The polynomial weight function attempts to produce a reasonable average weight by size class for all cases but further work is necessary to understand this relationship.

Improvements are expected after performing additional sieving experiments with a much finer sieving, using consistent decks for all products, such as 0, 12, 20, 25, 31, $37,40,45,50,63,75$ etc. instead of changing the decks as was done here. This sort of high resolution sieving will be carried out in an upcoming measurement and sieving trial to collect the necessary field measurements so that we can more accurately represent fragment weight variations by size class.

\section{ACKNOWLEDGEMENTS}

Thank you to ProcessIT Innovations for their continuing support in developing and intiating industry projects. Thank you to the MinBaS II research program and Nordkalk for their participation and support, and a thank you to our meaurement technology partners, MBV-Systems.

\section{REFERENCES}

Carlsson, O. and Nyberg, L. (1983). A method for estimation of fragmentation size distribution with automatic image processing. In Proceedings of the First International Symposium on Rock Fragmentation by Blasting FRAGBLAST, 333-345. Luleå, Sweden. 


\section{Sieve Size Distribution 606 measurements (5 sample average)}

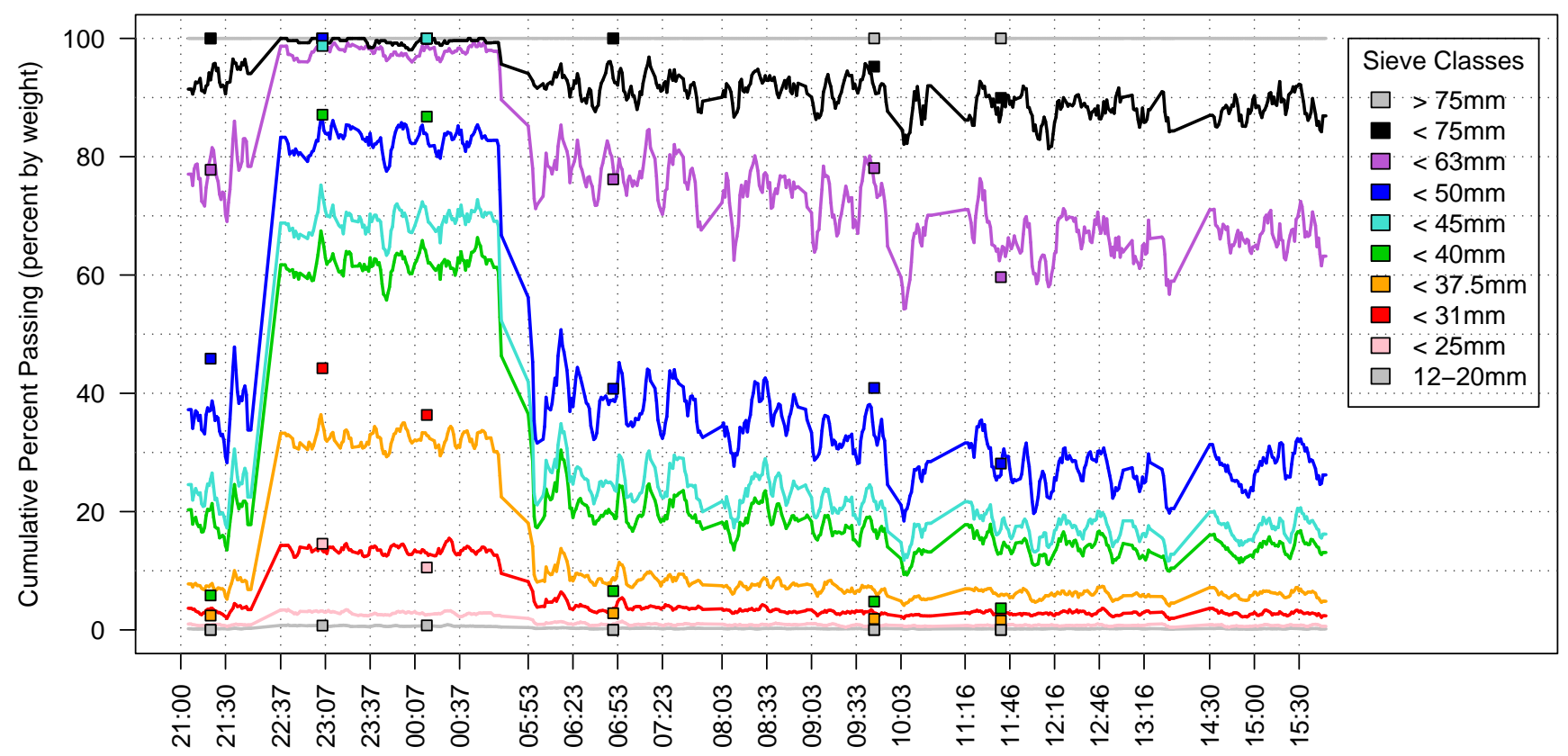

Fig. 7. Imaging and sieving results for 606 measurements over 10 hours of loading.

Frydendal, I. and Jones, R. (1998). Segmentation of sugar beets using image and graph processing. In ICPR 98 Proceedings $-14^{\text {th }}$ International Conference on Pattern Recognition, volume II, 16-20. Brisbane, Australia.

Kim, H., Haas, C., Rauch, A., and Browne, C. (2003). 3d image segmentation of aggregates from laser profiling. Computer Aided Civil and Infrastructure Engineering, 254-263.

Lee, J., Smith, M., Smith, L., and Midha, P. (2005). A mathematical morphology approach to image based $3 \mathrm{~d}$ particle shape analysis. In Machine Vision and Applications, volume 16(5), 282-288. Springer-Verlag.

Nelder, J. and Mead, R. (1965). A simplex method for function minimisation. The Computer Journal, 7, 308313.

Noy, M.J. (2006). The latest in on-line fragmentation measurement - stereo imaging over a conveyor. In Proceedings of the Eighth International Symposium on Rock Fragmentation by Blasting - FRAGBLAST 8, 6166.

Potts, G. and Ouchterlony, F. (2005). The capacity of image analysis to measure fragmentation, an evaluation using split desktop. Technical report, Swebrec - Swedish Rock Breaking Institute. ISSSN 1653-5006.

Rosato, A., Strandburg, K., Prinz, F., and Swendsen, R. (1987). Why the brazil nuts are on top: Size segregation of particulate matter by shaking. Physical Review Letters, 58(10).

Thurley, M.J. (2002). Three Dimensional Data Analysis for the Separation and Sizing of Rock Piles in Mining. Ph.D. thesis, Monash University. URL http://image3d6.eng.monash.edu.au/thesis.html.

Thurley, M.J. (2009). Fragmentation size measurement using 3d surface imaging. In Proceedings of the Ninth
International Symposium on Rock Fragmentation by Blasting - FRAGBLAST 9. In submission.

Thurley, M. and Andersson, T. (2007). An industrial 3d vision system for size measurement of iron ore green pellets using morphological image segmentation. Minerals Engineering, 21(5), 405-415. URL http://dx.doi .org/10.1016/j .mineng. 2007.10.020.

Thurley, M. and Ng, K. (2005). Identifying, visualizing, and comparing regions in irregularly spaced 3d surface data. Computer Vision and Image Understanding, 98(2), 239-270. URL http://dx.doi.org/10.1016/j.cviu.2003.12.002.

Thurley, M. and Ng, K. (2008). Identification and sizing of the entirely visible rocks from segmented $3 \mathrm{~d}$ surface data of laboratory rock piles. Computer $\mathrm{Vi}$ sion and Image Understanding, 111(2), 170-178. URL http://dx.doi.org/10.1016/j.cviu.2007.09.009.

Wang, W. (2006). Image analysis of particles by modified ferret method - best-fit rectangle. Power Technology, $1-10$. 